\title{
Existence of mild solutions to semilinear fractional differential inclusion with deviated advanced nonlocal conditions
}

\author{
Mohamed A. E. Herzallah* and Ashraf H. A. Radwan
}

*Correspondence:

m_herzallah75@hotmail.com

Faculty of Science, Zagazig

University, Zagazig, Egypt

\begin{abstract}
This paper is devoted to study the existence of at least one continuous mild solution to semilinear fractional differential inclusion with deviated-advanced nonlocal conditions. We develop the results obtained, by exchanging the deviated-advanced nonlocal condition to an integral form. Our results will be accomplished by using the nonlinear Leray-Schauder's alternative fixed point theorem. The main results are well illustrated with the aid of an example.
\end{abstract}

Keywords: Caputo derivative, Differential inclusion, Nonlocal conditions, Deviated-advanced nonlocal condition, Schauder's fixed point theorem

AMS classification: $26 \mathrm{~A} 33-28 \mathrm{~B} 99-34 \mathrm{G} 20$ - 45N05

\section{Introduction}

In the last two decades, fractional differential equations have become one of the most important branches in mathematics, due to its wide range of applications in different fields of sciences and engineering, such as in physics, chemistry, and biology [1-7].

Over the decades, a number of definitions of fractional derivatives are presented such as the most prevalent definitions of Riemann-Liouville and Caputo. Differential inclusions established as a part of the general theory of differential equations and penetrated different areas of sciences as a consequence of their numerous applications [8-19].

Many authors are interested in studying different classes of differential inclusions by using several forms of accompanying conditions. Nonlocal conditions have a leading role in describing some peculiarities of chemical, biological, physical, or other processes occurring at various positions inside the domain, which is clearly not possible with the end-point (initial/boundary) conditions. In fact, nonlocal conditions give more precise measurements, accurate results, and better effect than the classical conditions. For instance, to describe the diffusion phenomenon of a small amount of gas in a transparent tube, Deng [20] used the final nonlocal condition $u(x, T)-G u(x)=\sum_{j=1}^{m} b_{j} u\left(x, t_{j}\right)$, where $G$ is given function and $t_{j} \in J$ and $b_{j}$ are real numbers. Also, the integral boundary conditions have various applications in blood flow problems, theorem-elasticity, chemical engineering, populations dynamics, etc. [8, 21]. 
Chang and Nieto [12] studied the existence of solutions for the class of fractional differential inclusion

$$
{ }^{c} D^{\delta} y(t) \in F(t, y(t)), t \in[0,1]
$$

with the boundary conditions $y(0)=\alpha, y(1)=\beta, \alpha, \beta \neq 0$, where ${ }^{c} D^{\delta}$ is the Caputo fractional derivative of order $\delta \in(1,2)$.

Lian et al. [22] discussed the existence of mild solutions for the fractional differential inclusions

$$
{ }^{c} D^{q} u(t) \in A u(t)+F(t, u(t)), q \in(0,1), t \in[0, b]
$$

with the nonlocal condition

$$
u(0)=g(u),
$$

where $A: D(A) \rightarrow X$ is the infinitesimal generator of a strongly continuous semigroup $\{T(t)\}_{t \geq 0}$ on $X, F$ is an upper-Carathéodory set-valued function, and the nonlocal term $g$ is a given function from $C(J, X)$ into $X$.

Wang et al. [16] gave two existence results to the nonlocal semilinear differential inclusions with fractional order

$$
{ }^{c} D^{q} u(t) \in A u(t)+F(t, u(t)), q \in(0,1), t \in[0,1]
$$

with the nonlocal condition

$$
u(0)=\sum_{k=1}^{m} a_{k} u\left(t_{k}\right), \quad 0=t_{0}<t_{1} \leq t_{2} \leq \ldots \leq t_{m}=1,
$$

when the nonlinear term $F:[0,1] \times E \rightarrow P(E)-\{\phi\}$ is a multifunction, $E$ is a separable Banach space and $\mathrm{A}$ is a bounded operator.

El-Sayed et al. [23] discussed the existence of solutions to the differential inclusion

$$
x^{\prime}(t) \in F(t, x(t)) \text {, a.e, } t \in(0,1)
$$

under the new nonlocal conditions, the deviated-advanced nonlocal condition,

$$
\sum_{k=1}^{m} a_{k} x\left(\phi\left(\tau_{k}\right)\right)=\alpha \sum_{j=1}^{n} b_{j} x\left(\psi\left(\eta_{j}\right)\right), a_{k}, b_{j}>0 .
$$

where $F\left(.\right.$, . ) is a set-valued function from $[0,1] \times \mathbb{R}$ into $P\left(\mathbb{R}^{+}\right)$(the power set of $\mathbb{R}^{+}$), $\alpha>$ 0 is a parameter, $\tau_{k}, \eta_{j} \in(0,1)$, and $\phi, \psi$ are, respectively, deviated and advanced given functions. In [23], some special cases of nonlocal and integral conditions are displayed such as $\sum_{k=1}^{m} a_{k} x\left(\phi\left(\tau_{k}\right)\right)=0$ where $\tau_{k} \in(0,1), \sum_{k=1}^{m} a_{k} x\left(\phi\left(\tau_{k}\right)\right)=\alpha x(\psi(\eta))$, where $\tau_{k}, \eta \in(0,1)$ and $\int_{0}^{1} x(\phi(s)) d s=\alpha \int_{0}^{1} x(\psi(s)) d s$.

The aim of this paper is to discuss the existence of solutions to the following class of deviated-advanced nonlocal semilinear fractional differential inclusions

$$
\left\{\begin{array}{l}
{ }^{c} D^{\alpha} u(t)-A(t) u(t) \in F(t, u(t)), \quad \text { a.e, } \quad t \in J:=[0, T], T<\infty ; \\
\sum_{k=1}^{m} a_{k} u\left(\phi\left(\tau_{k}\right)\right)=\lambda \sum_{j=1}^{n} b_{j} u\left(\psi\left(\eta_{j}\right)\right), a_{k}, b_{j}>0 .
\end{array}\right.
$$

where $F: J \times \mathbb{R} \rightarrow P\left(\mathbb{R}^{+}\right)$is $L^{1}$-Carathéodory set-valued function, $\tau_{k}, \eta_{j} \in J, \lambda>0$ is a parameter, and $\phi, \psi$ are, respectively, deviated and advanced given functions. ${ }^{c} D^{\alpha}$ denotes the Caputo fractional derivative of order $\alpha \in(0,1)$, and $A(t)$ is a bounded linear operator on a Banach space $X$ with domain $D(A) \subset X$. We develop our results to the following class of nonlocal problems with deviated-advanced integral conditions 


$$
\left\{\begin{array}{l}
{ }^{c} D^{\alpha} u(t)-A(t) u(t) \in F(t, u(t)), \quad \text { a.e, } \quad t \in J \\
\int_{0}^{T} u(\phi(s)) d s=\lambda \int_{0}^{T} u(\psi(s)) d s,
\end{array}\right.
$$

This paper is organized as follows: In the "Preliminaries" section, we display some preliminary definitions and theorems which are used throughout this paper. In the "Existence results" section, we study the existence of at least one continuous mild solution to Problem (1) by applying the nonlinear alternative fixed point theorem of LeraySchauder type, and we promote our outcomes to Problem (2) by giving an example to illustrate our results.

\section{Preliminaries}

Here, we introduce the main definitions and theorems which are used throughout this paper.

As usual, let $\mathbb{R}$ be the set of all real numbers and $\mathbb{N}$ be the set of all positive integers. For a Banach space $\left(X,\|\cdot\|_{X}\right)$, let $P(X)$ be the family of all subsets of $X, B(X)$ be the class of all bounded linear operators on $X$ with the norm $\|S\|_{B}:=\sup \{\|S v\|:\|v\|=1\}, C(J, X)$ be the set of all continuous functions $u: J \rightarrow X$ with the norm $\|u\|_{C}:=\sup \{\|u(t)\|: u \in$ $C(J, X), t \in J\}, C^{n}(J, X)$ be the set of all $n$-differentiable functions with $u^{(n)} \in C(J, X), n \in$ $\mathbb{N}, A C(J, X)$ be the set of all absolutely continuous functions from $J$ into $X$, and $L^{1}(J, X)$ be the class of Lebesgue integrable functions $v: J \rightarrow X$ with the norm $\|v\|_{L^{1}}:=\int_{J}\|v(t)\| d t$.

Let $\phi: J \rightarrow J$ be a deviated continuous real valued function (i.e., $\phi(t) \leq t$ ) and $\psi: J \rightarrow J$ be an advanced continuous real valued function (i.e., $\psi(t) \geq t$ ). As an example of the deviated function $\phi$, we can take $\phi(t)=\theta t, \theta \in(0,1)$ or $\phi(t)=t-\theta, \theta \in J, t>\theta$. As an example of the advanced function $\psi$, we can consider $\psi(t)=\sigma t, \sigma>1$ or $\psi(t)=t+\theta, \theta \in J, t<\theta$.

Definition 1 ([4]) The fractional integral of order $\alpha>0$ with the lower limit 0 for a function $u$ is defined as

$$
I^{\alpha} u(t)=\frac{1}{\Gamma(\alpha)} \int_{0}^{t}(t-s)^{\alpha-1} u(s) d s, t>0 .
$$

provided that the right-hand side is pointwise defined on $(0, \infty)$, where $\Gamma($.$) is the gamma$ function.

Definition 2 ([4]) The Caputo fractional derivative of order $\alpha$ where $0<\alpha<1$ with the lower limit of for a function $u \in A C(J, X)$ is defined as

$$
{ }^{c} D^{\alpha} u(t)=I^{1-\alpha} D^{1} u(t), t>0, D^{1}=d / d t .
$$

Define $P_{b d}(X):=\{W \in P(X): W$ is bounded $\}, P_{c p}(X):=\{W \in P(X)$ : $W$ is compact $\}, P_{c v}(X):=\{W \in P(X): W$ is convex $\}$, and $P_{c p, c v}(X):=\{W \in P(X)$ : $W$ is compact and convex\}.

For two sets $Z$ and $Y$, a (set-valued) function $F: Z \rightarrow P(Y)$ assigns to each $z \in Z$ some $F(z) \subseteq Y$. A fixed point of $F$ is a point $u \in Z$ such that $u \in F(u)$. The graph of $F$ is $G(F):=\{(z, y) \in Z \times Y ; y \in F(z)\}$. A function $f: Z \rightarrow Y$ is said to be a selection of $F$ if $G(f) \subseteq G(F)$.

A set-valued function $F: X \rightarrow P(X)$ is convex (closed) valued if $F(x)$ is convex (closed) for all $x \in X . F$ is bounded on bounded sets if $F(W):=\cup_{x \in W} F(x)$ is bounded in $X$ for 
all $W \in P_{b d}(X)$, that is, $\sup _{x \in W}\{\sup \{|z|: z \in F(x)\}\}<\infty$. $F$ is said to be completely continuous if $F(W)$ is relatively compact for every $W \in P_{b d}(X)$. If $F$ is completely continuous with nonempty compact values, then $F$ is upper semicontinuous if and only if $G(F)$ is closed. For more details, see [10, 24].

Definition 3 ([25]) A set-valued function $F: J \times \mathbb{R} \rightarrow P(\mathbb{R})$ is called $L^{1}$-Carathéodory if

(a) $t \rightarrow F(t, u)$ is measurable for each $u \in \mathbb{R}$;

(b) $u \rightarrow F(t, u)$ is upper semicontinuous for almost all $t \in J$;

(c) For each $r>0$, there exists $\varkappa_{r} \in L^{1}(J, \mathbb{R})$ such that

$$
\|F(t, u)\|=\sup \{|v|: v \in F(t, u)\} \leq \varkappa_{r}(t) \text { for a.e } t \in J \text { and all }|u| \leq r .
$$

Definition $4([26])$ For each $u \in C(J, \mathbb{R})$, define the set of $L^{1}$-selections of $F$ by

$$
S_{F, u}^{1}:=\left\{f \in L^{1}(J, \mathbb{R}): f(t) \in F(t, u(t)) \text { for a.e. } t \in J\right\} \text {. }
$$

Theorem 1 ([26]) The set of selections $S_{F, u}^{1}$ is nonempty for any $L^{1}$-Carathéodory setvalued function $F: J \times \mathbb{R} \rightarrow P(\mathbb{R})$ for each $u \in C(J, \mathbb{R})$.

Theorem $2([25,27])$ Let $X$ be a Banach space, $M$ be a closed convex subset of $X, \Lambda$ be an open subset of $M$ and $0 \in \Lambda$. Suppose that $F: \bar{\Lambda} \rightarrow P_{c p, c v}(M)$ is upper semicontinuous and compact map. Then, either

(i) F has a fixed point in $\bar{\Lambda}$, or

(ii) There is a $u \in \bar{\Lambda}$ and $\varrho \in(0,1)$ with $u \in \varrho F u$.

Lemma 1 ([26]) Let $X$ be a Banach space, $F: J \times \mathbb{R} \rightarrow P_{c p, c v}(X)$ be an $L^{1}$-Carathéodory set-valued map, and $\Omega: L^{1}(J, X) \rightarrow C(J, X)$ be a linear continuous map. Then, the operator

$$
\Omega o S_{F}^{1}: C(J, X) \rightarrow P_{c p, c v}(C(J, X)), u \mapsto \Omega\left(S_{F, u}^{1}\right)
$$

is a closed graph operator in $C(J, X) \times C(J, X)$.

\section{Existence results}

Consider the following assumptions:

$\left(H_{1}\right) A(t)$ is a bounded linear operator on $\mathbb{R}$ where $t \rightarrow A(t)$ is continuous in the strong operator topology and $N=\max \{|A(t)|: t \in J\}$.

$\left(H_{2}\right) \quad F: J \times \mathbb{R} \rightarrow P(\mathbb{R})$ is $L^{1}$-Carathéodory and has nonempty, compact, and convex values;

$\left(H_{3}\right) \quad \phi: J \rightarrow J, \phi(t) \leq t$ is a deviated continuous function.

$\left(H_{4}\right) \quad \psi: J \rightarrow J, \psi(t) \geq t$ is an advanced continuous function.

$\left(H_{5}\right)$ There exists a constant $v>0$ such that

$$
\frac{v \Gamma(1+\alpha)}{T^{\alpha}\left(v N+\|\varkappa\|_{L^{1}}\right)\left[1+|\gamma|\left(|\lambda| \sum_{j=1}^{n} b_{j}+\sum_{k=1}^{m} a_{k}\right)\right]}>1 .
$$

where $\gamma:=\left(\sum_{k=1}^{m} a_{k}-\lambda \sum_{j=1}^{n} b_{j}\right)^{-1}$.

We need the following lemma to define a mild solution for Problem (1). 
Lemma 2 Let $\sum_{k=1}^{m} a_{k} \neq \lambda \sum_{j=1}^{n} b_{j}$. For a given single-valued function $\mu \in C(J, \mathbb{R})$, the solution of the nonlocal problem

$$
\left\{\begin{array}{l}
{ }^{c} D^{\alpha} u(t)=A(t) u(t)+\mu(t) \text { a.e. } t \in J \\
\sum_{k=1}^{m} a_{k} u\left(\phi\left(\tau_{k}\right)\right)=\lambda \sum_{j=1}^{n} b_{j} u\left(\psi\left(\eta_{j}\right)\right), a_{k}, b_{j}>0 .
\end{array}\right.
$$

can be expressed by the integral equation

$$
\begin{aligned}
u(t)= & \gamma \lambda \sum_{j=1}^{n} b_{j} \int_{0}^{\psi\left(\eta_{j}\right)} \frac{\left(\psi\left(\eta_{j}\right)-s\right)^{\alpha-1}}{\Gamma(\alpha)} A(s) u(s) d s+\gamma \lambda \sum_{j=1}^{n} b_{j} \int_{0}^{\psi\left(\eta_{j}\right)} \frac{\left(\psi\left(\eta_{j}\right)-s\right)^{\alpha-1}}{\Gamma(\alpha)} \mu(s) d s \\
& -\gamma \sum_{k=1}^{m} a_{k} \int_{0}^{\phi\left(\tau_{k}\right)} \frac{\left(\phi\left(\tau_{k}\right)-s\right)^{\alpha-1}}{\Gamma(\alpha)} A(s) u(s) d s-\gamma \sum_{k=1}^{m} a_{k} \int_{0}^{\phi\left(\tau_{k}\right)} \frac{\left(\phi\left(\tau_{k}\right)-s\right)^{\alpha-1}}{\Gamma(\alpha)} \mu(s) d s \\
& +\int_{0}^{t} \frac{(t-s)^{\alpha-1}}{\Gamma(\alpha)} A(s) u(s) d s+\int_{0}^{t} \frac{(t-s)^{\alpha-1}}{\Gamma(\alpha)} \mu(s) d s .
\end{aligned}
$$

Proof Let $u(t)$ be a solution for Problem (5). Operating $I^{\alpha}$ on both sides of the fractional differential equation of Problem (5), we get

$$
u(t)=u(0)+\int_{0}^{t} \frac{(t-s)^{\alpha-1}}{\Gamma(\alpha)} A(s) u(s) d s+\int_{0}^{t} \frac{(t-s)^{\alpha-1}}{\Gamma(\alpha)} \mu(s) d s .
$$

Putting $t=\phi\left(\tau_{k}\right)$ in (7), we obtain

$$
u\left(\phi\left(\tau_{k}\right)\right)=u(0)+\int_{0}^{\phi\left(\tau_{k}\right)} \frac{\left(\phi\left(\tau_{k}\right)-s\right)^{\alpha-1}}{\Gamma(\alpha)} A(s) u(s) d s+\int_{0}^{\phi\left(\tau_{k}\right)} \frac{\left(\phi\left(\tau_{k}\right)-s\right)^{\alpha-1}}{\Gamma(\alpha)} \mu(s) d s,
$$

then

$$
\begin{aligned}
\sum_{k=1}^{m} a_{k} u\left(\phi\left(\tau_{k}\right)\right)= & \sum_{k=1}^{m} a_{k} u(0)+\sum_{k=1}^{m} a_{k} \int_{0}^{\phi\left(\tau_{k}\right)} \frac{\left(\phi\left(\tau_{k}\right)-s\right)^{\alpha-1}}{\Gamma(\alpha)} A(s) u(s) d s \\
& +\sum_{k=1}^{m} a_{k} \int_{0}^{\phi\left(\tau_{k}\right)} \frac{\left(\phi\left(\tau_{k}\right)-s\right)^{\alpha-1}}{\Gamma(\alpha)} \mu(s) d s
\end{aligned}
$$

Putting $t=\psi\left(\eta_{j}\right)$ in (7), we obtain

$$
u\left(\psi\left(\eta_{j}\right)\right)=u(0)+\int_{0}^{\psi\left(\eta_{j}\right)} \frac{\left(\psi\left(\eta_{j}\right)-s\right)^{\alpha-1}}{\Gamma(\alpha)} A(s) u(s) d s+\int_{0}^{\psi\left(\eta_{j}\right)} \frac{\left(\psi\left(\eta_{j}\right)-s\right)^{\alpha-1}}{\Gamma(\alpha)} \mu(s) d s,
$$

then

$$
\begin{aligned}
\lambda \sum_{j=1}^{n} b_{j} u\left(\psi\left(\eta_{j}\right)\right)= & \lambda \sum_{j=1}^{n} b_{j} u(0)+\lambda \sum_{j=1}^{n} b_{j} \int_{0}^{\psi\left(\eta_{j}\right)} \frac{\left(\psi\left(\eta_{j}\right)-s\right)^{\alpha-1}}{\Gamma(\alpha)} A(s) u(s) d s \\
& +\lambda \sum_{j=1}^{n} b_{j} \int_{0}^{\psi\left(\eta_{j}\right)} \frac{\left(\psi\left(\eta_{j}\right)-s\right)^{\alpha-1}}{\Gamma(\alpha)} \mu(s) d s .
\end{aligned}
$$

From (8), (9), and the nonlocal condition of Problem (5), we get

$$
\begin{aligned}
u(0)= & \gamma \lambda \sum_{j=1}^{n} b_{j} \int_{0}^{\psi\left(\eta_{j}\right)} \frac{\left(\psi\left(\eta_{j}\right)-s\right)^{\alpha-1}}{\Gamma(\alpha)} A(s) u(s) d s+\gamma \lambda \sum_{j=1}^{n} b_{j} \int_{0}^{\psi\left(\eta_{j}\right)} \frac{\left(\psi\left(\eta_{j}\right)-s\right)^{\alpha-1}}{\Gamma(\alpha)} \mu(s) d s \\
& -\gamma \sum_{k=1}^{m} a_{k} \int_{0}^{\phi\left(\tau_{k}\right)} \frac{\left(\phi\left(\tau_{k}\right)-s\right)^{\alpha-1}}{\Gamma(\alpha)} A(s) u(s) d s-\gamma \sum_{k=1}^{m} a_{k} \int_{0}^{\phi\left(\tau_{k}\right)} \frac{\left(\phi\left(\tau_{k}\right)-s\right)^{\alpha-1}}{\Gamma(\alpha)} \mu(s) d s .
\end{aligned}
$$

Substituting (10) into (7), we get the required. 
Definition 5 A function $u \in C(J, \mathbb{R})$ is said to be a mild solution for Problem (1), if

$$
\sum_{k=1}^{m} a_{k} u\left(\phi\left(\tau_{k}\right)\right)=\lambda \sum_{j=1}^{n} b_{j} u\left(\psi\left(\eta_{j}\right)\right), a_{k}, b_{j}>0
$$

and there exists a function $f \in L^{1}(J, \mathbb{R})$ such that $f(t) \in F(t, u(t))$ a.e. on $J$, and $u(t)$ satisfies the integral equation

$$
\begin{aligned}
u(t)= & \gamma \lambda \sum_{j=1}^{n} b_{j} \int_{0}^{\psi\left(\eta_{j}\right)} \frac{\left(\psi\left(\eta_{j}\right)-s\right)^{\alpha-1}}{\Gamma(\alpha)} A(s) u(s) d s+\gamma \lambda \sum_{j=1}^{n} b_{j} \int_{0}^{\psi\left(\eta_{j}\right)} \frac{\left(\psi\left(\eta_{j}\right)-s\right)^{\alpha-1}}{\Gamma(\alpha)} f(s) d s \\
& -\gamma \sum_{k=1}^{m} a_{k} \int_{0}^{\phi\left(\tau_{k}\right)} \frac{\left(\phi\left(\tau_{k}\right)-s\right)^{\alpha-1}}{\Gamma(\alpha)} A(s) u(s) d s-\gamma \sum_{k=1}^{m} a_{k} \int_{0}^{\phi\left(\tau_{k}\right)} \frac{\left(\phi\left(\tau_{k}\right)-s\right)^{\alpha-1}}{\Gamma(\alpha)} f(s) d s \\
& +\int_{0}^{t} \frac{(t-s)^{\alpha-1}}{\Gamma(\alpha)} A(s) u(s) d s+\int_{0}^{t} \frac{(t-s)^{\alpha-1}}{\Gamma(\alpha)} f(s) d s .
\end{aligned}
$$

In view of the nonlinear alternative fixed point theorem of Leray-Schauder type (Theorem 2), we will discuss the existence of at least one mild solution for Problem (1).

Theorem 3 Let the assumptions $\left(H_{1}\right)-\left(H_{5}\right)$ be satisfied and $\sum_{k=1}^{m} a_{k} \neq \lambda \sum_{j=1}^{n} b_{j}$. Then, Problem (1) has at least one mild solution $u \in C(J, \mathbb{R})$.

Proof Since assumption $\left(H_{2}\right)$ is satisfied, then by applying Theorem 1 , there exists a single-valued selection $f \in S_{F, u}^{1}$. So, consider the set-valued operator $K: C(J, \mathbb{R}) \rightarrow$ $P(C(J, \mathbb{R}))$ such that

$$
K u(t)=\left\{g \in C(J, \mathbb{R}): g(t)=\left\{\begin{array}{l}
\gamma \lambda \sum_{j=1}^{n} b_{j} \int_{0}^{\psi\left(\eta_{j}\right)} \frac{\left(\psi\left(\eta_{j}\right)-s\right)^{\alpha-1}}{\Gamma(\alpha)} A(s) u(s) d s \\
+\gamma \lambda \sum_{j=1}^{n} b_{j} \int_{0}^{\psi\left(\eta_{j}\right)} \frac{\left(\psi\left(\eta_{j}\right)-s\right)^{\alpha-1}}{\Gamma(\alpha)} f(s) d s \\
-\gamma \sum_{k=1}^{m} a_{k} \int_{0}^{\phi\left(\tau_{k}\right)} \frac{\left(\phi\left(\tau_{k}\right)-s\right)^{\alpha-1}}{\Gamma(\alpha)} A(s) u(s) d s \quad, f \in S_{F, u}^{1} \\
-\gamma \sum_{k=1}^{m} a_{k} \int_{0}^{\phi\left(\tau_{k}\right)} \frac{\left(\phi\left(\tau_{k}\right)-s\right)^{\alpha-1}}{\Gamma(\alpha)} f(s) d s \\
+\int_{0}^{t} \frac{(t-s)^{\alpha-1}}{\Gamma(\alpha)} A(s) u(s) d s+\int_{0}^{t} \frac{(t-s)^{\alpha-1}}{\Gamma(\alpha)} f(s) d s
\end{array}\right\} .\right.
$$

That is, for each $g \in K u, u \in C(J, \mathbb{R})$, there exists $f \in S_{F, u}^{1}$ such that

$$
\begin{aligned}
g(t)= & \gamma \lambda \sum_{j=1}^{n} b_{j} \int_{0}^{\psi\left(\eta_{j}\right)} \frac{\left(\psi\left(\eta_{j}\right)-s\right)^{\alpha-1}}{\Gamma(\alpha)} A(s) u(s) d s+\gamma \lambda \sum_{j=1}^{n} b_{j} \int_{0}^{\psi\left(\eta_{j}\right)} \frac{\left(\psi\left(\eta_{j}\right)-s\right)^{\alpha-1}}{\Gamma(\alpha)} f(s) d s \\
& -\gamma \sum_{k=1}^{m} a_{k} \int_{0}^{\phi\left(\tau_{k}\right)} \frac{\left(\phi\left(\tau_{k}\right)-s\right)^{\alpha-1}}{\Gamma(\alpha)} A(s) u(s) d s-\gamma \sum_{k=1}^{m} a_{k} \int_{0}^{\phi\left(\tau_{k}\right)} \frac{\left(\phi\left(\tau_{k}\right)-s\right)^{\alpha-1}}{\Gamma(\alpha)} f(s) d s \\
& +\int_{0}^{t} \frac{(t-s)^{\alpha-1}}{\Gamma(\alpha)} A(s) u(s) d s+\int_{0}^{t} \frac{(t-s)^{\alpha-1}}{\Gamma(\alpha)} f(s) d s .
\end{aligned}
$$

Now, we will show that $K$ satisfies the hypotheses of Theorem 2 .

The proof will be given in 4 steps:

Step 1: $(K$ is convex for each $u \in C(J, \mathbb{R}))$ 
Using assumption $\left(H_{2}\right)$, we have $F$ has a convex values, then the set of selections $S_{F, u}^{1}$ is convex. Therefore, the step is obvious.

Step 2: ( $K$ is a completely continuous operator)

Firstly, we have to show that $K$ maps bounded sets into bounded sets in $C(J, \mathbb{R})$.

For a positive number $r$, let $B_{r} \subset C(J, \mathbb{R})$ be defined as

$$
B_{r}=\{u \in C(J, \mathbb{R}):\|u\| \leq r\} .
$$

Clearly, $B_{r}$ is a bounded ball in $C(J, \mathbb{R})$.

Using (13) with applying $\left(H_{3}\right)$ and $\left(H_{4}\right)$, we get

$$
\begin{aligned}
g(t) \leq & \gamma \lambda \sum_{j=1}^{n} b_{j} \int_{0}^{\eta_{j}} \frac{\left(\eta_{j}-s\right)^{\alpha-1}}{\Gamma(\alpha)} A(s) u(s) d s+\gamma \lambda \sum_{j=1}^{n} b_{j} \int_{0}^{\eta_{j}} \frac{\left(\eta_{j}-s\right)^{\alpha-1}}{\Gamma(\alpha)} f(s) d s \\
& -\gamma \sum_{k=1}^{m} a_{k} \int_{0}^{\tau_{k}} \frac{\left(\tau_{k}-s\right)^{\alpha-1}}{\Gamma(\alpha)} A(s) u(s) d s-\gamma \sum_{k=1}^{m} a_{k} \int_{0}^{\tau_{k}} \frac{\left(\tau_{k}-s\right)^{\alpha-1}}{\Gamma(\alpha)} f(s) d s \\
& +\int_{0}^{t} \frac{(t-s)^{\alpha-1}}{\Gamma(\alpha)} A(s) u(s) d s+\int_{0}^{t} \frac{(t-s)^{\alpha-1}}{\Gamma(\alpha)} f(s) d s,
\end{aligned}
$$

then for $u \in B_{r}$,

$$
\begin{aligned}
\|g\| \leq & \left(r N+\|\varkappa\|_{L^{1}}\right)|\lambda \gamma| \sum_{j=1}^{n} b_{j} \int_{0}^{\eta_{j}} \frac{\left(\eta_{j}-s\right)^{\alpha-1}}{\Gamma(\alpha)} d s+\left(r N+\|\varkappa\|_{L^{1}}\right)|\gamma| \sum_{k=1}^{m} a_{k} \int_{0}^{\tau_{k}} \frac{\left(\tau_{k}-s\right)^{\alpha-1}}{\Gamma(\alpha)} d s \\
& +\left(r N+\|\varkappa\|_{L^{1}}\right) \int_{0}^{t} \frac{(t-s)^{\alpha-1}}{\Gamma(\alpha)} d s \\
\leq & \frac{T^{\alpha}\left(r N+\|\varkappa\|_{L^{1}}\right)}{\Gamma(1+\alpha)}\left[1+|\gamma|\left(|\lambda| \sum_{j=1}^{n} b_{j}+\sum_{k=1}^{m} a_{k}\right)\right] .
\end{aligned}
$$

Thus, $K$ maps bounded sets into bounded sets in $C(J, \mathbb{R})$.

Secondly, we have to prove that $K$ maps bounded sets into equicontinuous sets of $C(J, \mathbb{R})$.

Let $0<t_{1}<t_{2}<T$, for each $g \in K u$ and $u \in B_{r}$.

$$
\begin{aligned}
g\left(t_{2}\right)-g\left(t_{1}\right)= & \int_{0}^{t_{2}} \frac{\left(t_{2}-s\right)^{\alpha-1}}{\Gamma(\alpha)} A(s) u(s) d s-\int_{0}^{t_{1}} \frac{\left(t_{1}-s\right)^{\alpha-1}}{\Gamma(\alpha)} A(s) u(s) d s \\
& +\int_{0}^{t_{2}} \frac{\left(t_{2}-s\right)^{\alpha-1}}{\Gamma(\alpha)} f(s) d s-\int_{0}^{t_{1}} \frac{\left(t_{1}-s\right)^{\alpha-1}}{\Gamma(\alpha)} f(s) d s \\
= & \int_{t_{1}}^{t_{2}} \frac{\left(t_{2}-s\right)^{\alpha-1}}{\Gamma(\alpha)} A(s) u(s) d s+\int_{t_{1}}^{t_{2}} \frac{\left(t_{2}-s\right)^{\alpha-1}}{\Gamma(\alpha)} f(s) d s \\
& +\int_{0}^{t_{1}}\left(\frac{\left(t_{2}-s\right)^{\alpha-1}}{\Gamma(\alpha)}-\frac{\left(t_{1}-s\right)^{\alpha-1}}{\Gamma(\alpha)}\right) A(s) u(s) d s \\
& +\int_{0}^{t_{1}}\left(\frac{\left(t_{2}-s\right)^{\alpha-1}}{\Gamma(\alpha)}-\frac{\left(t_{1}-s\right)^{\alpha-1}}{\Gamma(\alpha)}\right) f(s) d s,
\end{aligned}
$$


then

$$
\begin{aligned}
\left|g\left(t_{2}\right)-g\left(t_{1}\right)\right| \leq & N \int_{t_{1}}^{t_{2}} \frac{\left(t_{2}-s\right)^{\alpha-1}}{\Gamma(\alpha)}|u(s)| d s+\int_{t_{1}}^{t_{2}} \frac{\left(t_{2}-s\right)^{\alpha-1}}{\Gamma(\alpha)}|f(s)| d s \\
& +N \int_{0}^{t_{1}}\left(\frac{\left(t_{2}-s\right)^{\alpha-1}}{\Gamma(\alpha)}-\frac{\left(t_{1}-s\right)^{\alpha-1}}{\Gamma(\alpha)}\right)|u(s)| d s \\
& +\int_{0}^{t_{1}}\left(\frac{\left(t_{2}-s\right)^{\alpha-1}}{\Gamma(\alpha)}-\frac{\left(t_{1}-s\right)^{\alpha-1}}{\Gamma(\alpha)}\right)|f(s)| d s \\
\leq & \left(r N+\|\varkappa\|_{L^{1}}\right)\left(\int_{t_{1}}^{t_{2}} \frac{\left(t_{2}-s\right)^{\alpha-1}}{\Gamma(\alpha)} d s+\int_{0}^{t_{1}}\left(\frac{\left(t_{2}-s\right)^{\alpha-1}}{\Gamma(\alpha)}-\frac{\left(t_{1}-s\right)^{\alpha-1}}{\Gamma(\alpha)}\right) d s\right) \\
\leq & \frac{1}{\Gamma(\alpha+1)}\left(r N+\|\mathcal{L}\|_{L^{1}}\right)\left|t_{2}^{\alpha}-t_{1}^{\alpha}\right| .
\end{aligned}
$$

Obviously, the right-hand side tends to 0 independently of $u \in B_{r}$ as $t_{2}$ tends to $t_{1}$. That is, $K$ maps bounded sets into equicontinuous sets of $C(J, \mathbb{R})$. Applying Arzela-Ascoli theorem, we have that $K$ is a completely continuous operator.

Step 3: ( $K$ is an upper semicontinuous operator)

From $\left(H_{2}\right)$ and step $2, K$ is completely continuous with nonempty compact values. So, we have to show that $K$ has a closed graph to be an upper semicontinuous operator.

Let $u_{n} \rightarrow \breve{u}, g_{n} \rightarrow \check{g}$ where $g_{n} \in K u_{n}$. We need to prove that $\breve{g} \in K \breve{u}$. Associated with $g_{n} \in K u_{n}$, there exists $f_{n} \in S_{F, u_{n}}^{1}$ such that for each $t \in J$, we have

$$
\begin{aligned}
g_{n}(t)= & \gamma \lambda \sum_{j=1}^{n} b_{j} \int_{0}^{\psi\left(\eta_{j}\right)} \frac{\left(\psi\left(\eta_{j}\right)-s\right)^{\alpha-1}}{\Gamma(\alpha)} A(s) u_{n}(s) d s+\gamma \lambda \sum_{j=1}^{n} b_{j} \int_{0}^{\psi\left(\eta_{j}\right)} \frac{\left(\psi\left(\eta_{j}\right)-s\right)^{\alpha-1}}{\Gamma(\alpha)} f_{n}(s) d s \\
& -\gamma \sum_{k=1}^{m} a_{k} \int_{0}^{\phi\left(\tau_{k}\right)} \frac{\left(\phi\left(\tau_{k}\right)-s\right)^{\alpha-1}}{\Gamma(\alpha)} A(s) u_{n}(s) d s-\gamma \sum_{k=1}^{m} a_{k} \int_{0}^{\phi\left(\tau_{k}\right)} \frac{\left(\phi\left(\tau_{k}\right)-s\right)^{\alpha-1}}{\Gamma(\alpha)} f_{n}(s) d s \\
& +\int_{0}^{t} \frac{(t-s)^{\alpha-1}}{\Gamma(\alpha)} A(s) u_{n}(s) d s+\int_{0}^{t} \frac{(t-s)^{\alpha-1}}{\Gamma(\alpha)} f_{n}(s) d s .
\end{aligned}
$$

We want to prove that there exists $\breve{f} \in S_{F, \breve{u}}^{1}$ such that for all $t \in J$, we have

$$
\begin{aligned}
\check{g}(t)= & \gamma \lambda \sum_{j=1}^{n} b_{j} \int_{0}^{\psi\left(\eta_{j}\right)} \frac{\left(\psi\left(\eta_{j}\right)-s\right)^{\alpha-1}}{\Gamma(\alpha)} A(s) \check{u}(s) d s+\gamma \lambda \sum_{j=1}^{n} b_{j} \int_{0}^{\psi\left(\eta_{j}\right)} \frac{\left(\psi\left(\eta_{j}\right)-s\right)^{\alpha-1}}{\Gamma(\alpha)} \check{f}(s) d s \\
& -\gamma \sum_{k=1}^{m} a_{k} \int_{0}^{\phi\left(\tau_{k}\right)} \frac{\left(\phi\left(\tau_{k}\right)-s\right)^{\alpha-1}}{\Gamma(\alpha)} A(s) \check{u}(s) d s-\gamma \sum_{k=1}^{m} a_{k} \int_{0}^{\phi\left(\tau_{k}\right)} \frac{\left(\phi\left(\tau_{k}\right)-s\right)^{\alpha-1}}{\Gamma(\alpha)} \check{f}(s) d s \\
& +\int_{0}^{t} \frac{(t-s)^{\alpha-1}}{\Gamma(\alpha)} A(s) \check{u}(s) d s+\int_{0}^{t} \frac{(t-s)^{\alpha-1}}{\Gamma(\alpha)} \check{f}(s) d s .
\end{aligned}
$$

In view of Lemma 1 , define the continuous linear operator $\Omega: L^{1}(J, \mathbb{R}) \rightarrow C(J, \mathbb{R})$ as

$$
\begin{aligned}
\Omega(f)(t)= & \gamma \lambda \sum_{j=1}^{n} b_{j} \int_{0}^{\psi\left(\eta_{j}\right)} \frac{\left(\psi\left(\eta_{j}\right)-s\right)^{\alpha-1}}{\Gamma(\alpha)} A(s) u(s) d s+\gamma \lambda \sum_{j=1}^{n} b_{j} \int_{0}^{\psi\left(\eta_{j}\right)} \frac{\left(\psi\left(\eta_{j}\right)-s\right)^{\alpha-1}}{\Gamma(\alpha)} f(s) d s \\
& -\gamma \sum_{k=1}^{m} a_{k} \int_{0}^{\phi\left(\tau_{k}\right)} \frac{\left(\phi\left(\tau_{k}\right)-s\right)^{\alpha-1}}{\Gamma(\alpha)} A(s) u(s) d s-\gamma \sum_{k=1}^{m} a_{k} \int_{0}^{\phi\left(\tau_{k}\right)} \frac{\left(\phi\left(\tau_{k}\right)-s\right)^{\alpha-1}}{\Gamma(\alpha)} f(s) d s \\
& +\int_{0}^{t} \frac{(t-s)^{\alpha-1}}{\Gamma(\alpha)} A(s) u(s) d s+\int_{0}^{t} \frac{(t-s)^{\alpha-1}}{\Gamma(\alpha)} f(s) d s .
\end{aligned}
$$


Thus,

$$
\begin{aligned}
\left|g_{n}(t)-\check{g}(t)\right| \leq & |\gamma \lambda| N \sum_{j=1}^{n} b_{j} \int_{0}^{\psi\left(\eta_{j}\right)} \frac{\left(\psi\left(\eta_{j}\right)-s\right)^{\alpha-1}}{\Gamma(\alpha)}\left|u_{n}(s)-\check{u}(s)\right| d s \\
& +|\gamma \lambda| \sum_{j=1}^{n} b_{j} \int_{0}^{\psi\left(\eta_{j}\right)} \frac{\left(\psi\left(\eta_{j}\right)-s\right)^{\alpha-1}}{\Gamma(\alpha)}\left|f_{n}(s)-\check{f}(s)\right| d s \\
& +|\gamma| N \sum_{k=1}^{m} a_{k} \int_{0}^{\phi\left(\tau_{k}\right)} \frac{\left(\phi\left(\tau_{k}\right)-s\right)^{\alpha-1}}{\Gamma(\alpha)}\left|u_{n}(s)-\check{u}(s)\right| d s \\
& +|\gamma| \sum_{k=1}^{m} a_{k} \int_{0}^{\phi\left(\tau_{k}\right)} \frac{\left(\phi\left(\tau_{k}\right)-s\right)^{\alpha-1}}{\Gamma(\alpha)}\left|f_{n}(s)-\check{f}(s)\right| d s \\
& +N \int_{0}^{t} \frac{(t-s)^{\alpha-1}}{\Gamma(\alpha)}\left|u_{n}(s)-\bar{u}(s)\right| d s+\int_{0}^{t} \frac{(t-s)^{\alpha-1}}{\Gamma(\alpha)}\left|f_{n}(s)-\check{f}(s)\right| d s .
\end{aligned}
$$

That is, $g_{n}(t) \rightarrow \check{g}(t)$ as $n \rightarrow \infty$. Applying Lemma 1 , we have $\Omega o S_{F, u}^{1}$ as a closed graph operator in $C(J, \mathbb{R}) \times C(J, \mathbb{R})$ and $g_{n} \in \Omega\left(S_{F, u_{n}}^{1}\right)$. Since $u_{n} \rightarrow \check{u}$, then we have $\check{g}(t)$ satisfies (18) for some $\breve{f} \in S_{F, \breve{u}}^{1}$. Therefore, $K$ is an upper semicontinuous operator.

Step 4: (There exists an open set $\Lambda \subset C(J, \mathbb{R})$ with $u \notin K u$ for $\varrho \in(0,1)$ and $u \in \partial \Lambda$ )

Let $\varrho \in(0,1)$ and $u \in \varrho K u$. Then, there exists $f \in L^{1}(J, \mathbb{R})$ with $f \in S_{F, u}^{1}$, such that, for $t \in J$, we have $g \in K u$ satisfies (13). As in proving step 2, we get

$$
\|g\| \leq \frac{T^{\alpha}\left(N\|u\|+\|\varkappa\|_{L^{1}}\right)}{\Gamma(1+\alpha)}\left[1+|\gamma|\left(|\lambda| \sum_{j=1}^{n} b_{j}+\sum_{k=1}^{m} a_{k}\right)\right] .
$$

Thus,

$$
\frac{\Gamma(1+\alpha)\|u\|}{T^{\alpha}\left(N\|u\|+\|\varkappa\|_{L^{1}}\right)\left[1+|\gamma|\left(|\lambda| \sum_{j=1}^{n} b_{j}+\sum_{k=1}^{m} a_{k}\right)\right]} \leq 1 .
$$

In view of $\left(H_{5}\right)$, there exists $v$ such that $v \neq\|u\|$. Set

$$
\Lambda:=\{u \in C(J, \mathbb{R}):\|u\|<v+1\} .
$$

From the definition of $\Lambda$, there is no $u \in \partial \Lambda$ such that $u \in \varrho K u$ for some $\varrho \in(0,1)$. Further, we have that the operator $K: \bar{\Lambda} \rightarrow P_{c p, c v}\left(B_{r}\right)$ is an upper semicontinuous and completely continuous operator.

Consequently, by the nonlinear alternative of Leray-Schauder type (Theorem 2), we deduce that $K$ has a fixed point $u \in \bar{\Lambda}$ which is a mild solution for Problem (1).

To prove the existence of at least one mild solution for Problem (2), we give the following lemma.

Lemma 3 Let $\sum_{k=1}^{m} a_{k} \neq \lambda \sum_{j=1}^{n} b_{j}$. For a given single-valued function $y \in C(J, \mathbb{R})$, the solution of the nonlocal problem

$$
\left\{\begin{array}{l}
{ }^{c} D^{\alpha} u(t)=A(t) u(t)+y(t) \text { a.e. } t \in J \\
\int_{0}^{T} u(\phi(s)) d s=\lambda \int_{0}^{T} u(\psi(s)) d s
\end{array}\right.
$$

can be expressed by the integral equation 


$$
\begin{aligned}
u(t)= & \beta \lambda \int_{0}^{T} \int_{0}^{\psi(s)} \frac{(\psi(s)-\theta)^{\alpha-1}}{\Gamma(\alpha)} A(\theta) u(\theta) d \theta d s+\beta \lambda \int_{0}^{T} \int_{0}^{\psi(s)} \frac{(\psi(s)-\theta)^{\alpha-1}}{\Gamma(\alpha)} y(\theta) d \theta d s \\
& -\beta \int_{0}^{T} \int_{0}^{\phi(s)} \frac{(\phi(s)-\theta)^{\alpha-1}}{\Gamma(\alpha)} A(\theta) u(\theta) d \theta d s-\beta \int_{0}^{T} \int_{0}^{\phi(s)} \frac{(\phi(s)-\theta)^{\alpha-1}}{\Gamma(\alpha)} y(\theta) d \theta d s \\
& +\int_{0}^{t} \frac{(t-\theta)^{\alpha-1}}{\Gamma(\alpha)} A(\theta) u(\theta) d \theta+\int_{0}^{t} \frac{(t-\theta)^{\alpha-1}}{\Gamma(\alpha)} y(\theta) d \theta
\end{aligned}
$$

where $\beta=\frac{1}{T(1-\lambda)}$ and $\lambda \neq 1$.

Proof Let $u(t)$ be a solution for Problem (21). Operating $I^{\alpha}$ on both sides of the fractional differential equation of Problem (21), we get

$$
u(t)=u(0)+\int_{0}^{t} \frac{(t-\theta)^{\alpha-1}}{\Gamma(\alpha)} A(\theta) u(\theta) d \theta+\int_{0}^{t} \frac{(t-\theta)^{\alpha-1}}{\Gamma(\alpha)} y(\theta) d \theta .
$$

Putting $t=\phi(s)$ in (23), we obtain

$$
u(\phi(s))=u(0)+\int_{0}^{\phi(s)} \frac{(\phi(s)-\theta)^{\alpha-1}}{\Gamma(\alpha)} A(\theta) u(\theta) d \theta+\int_{0}^{\phi(s)} \frac{(\phi(s)-\theta)^{\alpha-1}}{\Gamma(\alpha)} y(\theta) d \theta,
$$

then

$$
\begin{aligned}
\int_{0}^{T} u(\phi(s)) d s= & \int_{0}^{T} u(0) d s+\int_{0}^{T} \int_{0}^{\phi(s)} \frac{(\phi(s)-\theta)^{\alpha-1}}{\Gamma(\alpha)} A(\theta) u(\theta) d \theta d s \\
& +\int_{0}^{T} \int_{0}^{\phi(s)} \frac{(\phi(s)-\theta)^{\alpha-1}}{\Gamma(\alpha)} y(\theta) d \theta d s .
\end{aligned}
$$

Putting $t=\psi(s)$ in (23), we obtain

$$
u(\psi(s))=u(0)+\int_{0}^{\psi(s)} \frac{(\psi(s)-\theta)^{\alpha-1}}{\Gamma(\alpha)} A(\theta) u(\theta) d \theta+\int_{0}^{\psi(s)} \frac{(\psi(s)-\theta)^{\alpha-1}}{\Gamma(\alpha)} g(\theta, u(\theta)) d \theta,
$$

then

$$
\begin{aligned}
\lambda \int_{0}^{T} u(\psi(s)) d s= & \lambda \int_{0}^{T} u(0) d s+\lambda \int_{0}^{T} \int_{0}^{\psi(s)} \frac{(\psi(s)-\theta)^{\alpha-1}}{\Gamma(\alpha)} A(\theta) u(\theta) d \theta d s \\
& +\lambda \int_{0}^{T} \int_{0}^{\psi(s)} \frac{(\psi(s)-\theta)^{\alpha-1}}{\Gamma(\alpha)} y(\theta) d \theta d s, \lambda \neq 1 .
\end{aligned}
$$

From (24), (25), and the nonlocal condition of Problem (21), we get

$$
\begin{aligned}
u(0)= & \beta \lambda \int_{0}^{T} \int_{0}^{\psi(s)} \frac{(\psi(s)-\theta)^{\alpha-1}}{\Gamma(\alpha)} A(\theta) u(\theta) d \theta d s+\beta \lambda \int_{0}^{T} \int_{0}^{\psi(s)} \frac{(\psi(s)-\theta)^{\alpha-1}}{\Gamma(\alpha)} y(\theta) d \theta d s \\
& -\beta \int_{0}^{T} \int_{0}^{\phi(s)} \frac{(\phi(s)-\theta)^{\alpha-1}}{\Gamma(\alpha)} A(\theta) u(\theta) d \theta d s-\beta \int_{0}^{T} \int_{0}^{\phi(s)} \frac{(\phi(s)-\theta)^{\alpha-1}}{\Gamma(\alpha)} y(\theta) d \theta d s .
\end{aligned}
$$

where $\lambda \neq 1$ and $\beta=\frac{1}{T(1-\lambda)}$.

Substituting (26) into (23), we get (22). Then, the proof is completed.

Definition 6 A function $u \in C(J, \mathbb{R})$ is said to be a mild solution for Problem (2), if

$$
\int_{0}^{T} u(\phi(s)) d s=\lambda \int_{0}^{T} u(\psi(s)) d s,
$$


and there exists a function $f \in L^{1}(J, \mathbb{R})$ such that $f(t) \in F(t, u(t))$ a.e. on $J$, and $u(t)$ satisfies the integral equation

$$
\begin{aligned}
u(t)= & \beta \lambda \int_{0}^{T} \int_{0}^{\psi(s)} \frac{(\psi(s)-\theta)^{\alpha-1}}{\Gamma(\alpha)} A(\theta) u(\theta) d \theta d s+\beta \lambda \int_{0}^{T} \int_{0}^{\psi(s)} \frac{(\psi(s)-\theta)^{\alpha-1}}{\Gamma(\alpha)} f(\theta) d \theta d s \\
& -\beta \int_{0}^{T} \int_{0}^{\phi(s)} \frac{(\phi(s)-\theta)^{\alpha-1}}{\Gamma(\alpha)} A(\theta) u(\theta) d \theta d s-\beta \int_{0}^{T} \int_{0}^{\phi(s)} \frac{(\phi(s)-\theta)^{\alpha-1}}{\Gamma(\alpha)} f(\theta) d \theta d s \\
& +\int_{0}^{t} \frac{(t-\theta)^{\alpha-1}}{\Gamma(\alpha)} A(\theta) u(\theta) d \theta+\int_{0}^{t} \frac{(t-\theta)^{\alpha-1}}{\Gamma(\alpha)} f(\theta) d \theta .
\end{aligned}
$$

As in proving Theorem 3, we infer the existence of at least one mild solution for Problem (2).

Theorem 4 Let the assumptions $\left(H_{1}\right)-\left(H_{4}\right)$ be satisfied. Then, Problem (2) has at least one solution $u \in C(J, \mathbb{R})$ if there exists a positive constant $\varpi$ such that

$$
\frac{\varpi \Gamma(2+\alpha)}{T^{\alpha}(2+\alpha)\left(\varpi N+\|\varkappa\|_{L_{1}}\right)}>1
$$

Proof Since the assumption $\left(H_{2}\right)$ be satisfied, then by applying Theorem 1 , there exists a single-valued selection $f \in S_{F, u}^{1}$. So, consider the set-valued operator $Q: C(J, \mathbb{R}) \rightarrow$ $P(C(J, \mathbb{R}))$ such that

$$
Q u(t)=\left\{h \in C(J, \mathbb{R}): h(t)=\left\{\begin{array}{l}
\beta \lambda \int_{0}^{T} \int_{0}^{\psi(s)} \frac{(\psi(s)-\theta)^{\alpha-1}}{\Gamma(\alpha)} A(\theta) u(\theta) d \theta d s \\
+\beta \lambda \int_{0}^{T} \int_{0}^{\psi(s)} \frac{(\psi(s)-\theta)^{\alpha-1}}{\Gamma(\alpha)} f(\theta) d \theta d s \\
-\beta \int_{0}^{T} \int_{0}^{\phi(s)} \frac{(\phi(s)-\theta)^{\alpha-1}}{\Gamma(\alpha)} A(\theta) u(\theta) d \theta d s \\
-\beta \int_{0}^{T} \int_{0}^{\phi(s)} \frac{(\phi(s)-\theta)^{\alpha-1}}{\Gamma(\alpha)} f(\theta) d \theta d s \\
+\int_{0}^{t} \frac{(t-\theta)^{\alpha-1}}{\Gamma(\alpha)} A(\theta) u(\theta) d \theta+\int_{0}^{t} \frac{(t-\theta)^{\alpha-1}}{\Gamma(\alpha)} f(\theta) d \theta .
\end{array}, f \in S_{F, u}^{1}\right\} .\right.
$$

We have to show that $Q$ satisfies the hypotheses of Theorem 2 .

Step 1:

In view of $\left(H_{2}\right), F$ has convex values, then $S_{F, u}^{1}$ is a convex set. So, $Q$ is convex for each $u \in C(J, \mathbb{R})$.

Step 2:

Consider the bounded ball $B_{\sigma} \subset C(J, \mathbb{R})$ such that

$$
B_{\sigma}=\{u \in C(J, \mathbb{R}):\|u\| \leq \sigma, \sigma>0\} .
$$

Then, for each $h \in Q u, u \in B_{\sigma}$, there exists $f \in S_{F, u}^{1}$, such that

$$
\begin{aligned}
h(t)= & \beta \lambda \int_{0}^{T} \int_{0}^{\psi(s)} \frac{(\psi(s)-\theta)^{\alpha-1}}{\Gamma(\alpha)} A(\theta) u(\theta) d \theta d s+\beta \lambda \int_{0}^{T} \int_{0}^{\psi(s)} \frac{(\psi(s)-\theta)^{\alpha-1}}{\Gamma(\alpha)} f(\theta) d \theta d s \\
& -\beta \int_{0}^{T} \int_{0}^{\phi(s)} \frac{(\phi(s)-\theta)^{\alpha-1}}{\Gamma(\alpha)} A(\theta) u(\theta) d \theta d s-\beta \int_{0}^{T} \int_{0}^{\phi(s)} \frac{(\phi(s)-\theta)^{\alpha-1}}{\Gamma(\alpha)} f(\theta) d \theta d s \\
& +\int_{0}^{t} \frac{(t-\theta)^{\alpha-1}}{\Gamma(\alpha)} A(\theta) u(\theta) d \theta+\int_{0}^{t} \frac{(t-\theta)^{\alpha-1}}{\Gamma(\alpha)} f(\theta) d \theta .
\end{aligned}
$$


Applying $\left(H_{3}\right)$ and $\left(H_{4}\right)$,

$$
\begin{aligned}
h(t) \leq & \beta \lambda \int_{0}^{T} \int_{0}^{s} \frac{(s-\theta)^{\alpha-1}}{\Gamma(\alpha)} A(\theta) u(\theta) d \theta d s+\beta \lambda \int_{0}^{T} \int_{0}^{s} \frac{(s-\theta)^{\alpha-1}}{\Gamma(\alpha)} f(\theta) d \theta d s \\
& -\beta \int_{0}^{T} \int_{0}^{s} \frac{(s-\theta)^{\alpha-1}}{\Gamma(\alpha)} A(\theta) u(\theta) d \theta d s-\beta \int_{0}^{T} \int_{0}^{s} \frac{(s-\theta)^{\alpha-1}}{\Gamma(\alpha)} f(\theta) d \theta d s \\
& +\int_{0}^{t} \frac{(t-\theta)^{\alpha-1}}{\Gamma(\alpha)} A(\theta) u(\theta) d \theta+\int_{0}^{t} \frac{(t-\theta)^{\alpha-1}}{\Gamma(\alpha)} f(\theta) d \theta \\
\leq & \beta(\lambda-1) \int_{0}^{T} \int_{0}^{s} \frac{(s-\theta)^{\alpha-1}}{\Gamma(\alpha)} A(\theta) u(\theta) d \theta d s+\beta(\lambda-1) \int_{0}^{T} \int_{0}^{s} \frac{(s-\theta)^{\alpha-1}}{\Gamma(\alpha)} f(\theta) d \theta d s \\
& +\int_{0}^{t} \frac{(t-\theta)^{\alpha-1}}{\Gamma(\alpha)} A(\theta) u(\theta) d \theta+\int_{0}^{t} \frac{(t-\theta)^{\alpha-1}}{\Gamma(\alpha)} f(\theta) d \theta .
\end{aligned}
$$

For $u \in B_{r}$, we obtain

$$
\begin{aligned}
|h(t)| & \leq\left(\sigma N+\|\varkappa\|_{L_{1}}\right)\left(|\beta(\lambda-1)| \int_{0}^{T} \int_{0}^{s} \frac{(s-\theta)^{\alpha-1}}{\Gamma(\alpha)} d \theta d s+\int_{0}^{t} \frac{(t-\theta)^{\alpha-1}}{\Gamma(\alpha)} d \theta\right) \\
& \leq \frac{T^{\alpha}\left(\sigma N+\|\varkappa\|_{L_{1}}\right)}{\Gamma(1+\alpha)}\left(\frac{T|\beta(\lambda-1)|}{1+\alpha}+1\right) \\
& \leq \frac{T^{\alpha}(2+\alpha)\left(\sigma N+\|\varkappa\|_{L_{1}}\right)}{\Gamma(2+\alpha)} .
\end{aligned}
$$

Thus, $Q$ maps bounded sets into bounded sets in $C(J, \mathbb{R})$.

Let $0<t_{1}<t_{2}<T$, for each $h \in Q u$ and $u \in B_{\sigma}$. Using (30), as in proving (16), we get

$$
\left|h\left(t_{2}\right)-h\left(t_{1}\right)\right| \leq \frac{\sigma N+\|\varkappa\|_{L^{1}}}{\Gamma(\alpha+1)}\left|t_{2}^{\alpha}-t_{1}^{\alpha}\right| \rightarrow 0 \text { as } t_{2} \rightarrow t_{1} .
$$

Therefore, $Q$ maps bounded sets into equicontinuous sets of $C(J, \mathbb{R})$. Applying ArzelaAscoli theorem, we have that $Q$ is a completely continuous operator.

Step 3:

Let $u_{n} \rightarrow \check{u}, h_{n} \rightarrow \check{h}$ where $h_{n} \in Q u_{n}$. We have to prove that $\breve{h} \in Q \breve{u}$.

Associated with $h_{n} \in Q u_{n}$, there exists $f_{n} \in S_{F, u_{n}}^{1}$ such that for each $t \in J$, we have

$$
\begin{aligned}
h_{n}(t)= & \beta \lambda \int_{0}^{T} \int_{0}^{\psi(s)} \frac{(\psi(s)-\theta)^{\alpha-1}}{\Gamma(\alpha)} A(\theta) u_{n}(\theta) d \theta d s+\beta \lambda \int_{0}^{T} \int_{0}^{\psi(s)} \frac{(\psi(s)-\theta)^{\alpha-1}}{\Gamma(\alpha)} f_{n}(\theta) d \theta d s \\
& -\beta \int_{0}^{T} \int_{0}^{\phi(s)} \frac{(\phi(s)-\theta)^{\alpha-1}}{\Gamma(\alpha)} A(\theta) u_{n}(\theta) d \theta d s-\beta \int_{0}^{T} \int_{0}^{\phi(s)} \frac{(\phi(s)-\theta)^{\alpha-1}}{\Gamma(\alpha)} f_{n}(\theta) d \theta d s \\
& +\int_{0}^{t} \frac{(t-\theta)^{\alpha-1}}{\Gamma(\alpha)} A(\theta) u_{n}(\theta) d \theta+\int_{0}^{t} \frac{(t-\theta)^{\alpha-1}}{\Gamma(\alpha)} f_{n}(\theta) d \theta \\
\leq & \beta \lambda \int_{0}^{T} \int_{0}^{s} \frac{(s-\theta)^{\alpha-1}}{\Gamma(\alpha)} A(\theta) u_{n}(\theta) d \theta d s+\beta \lambda \int_{0}^{T} \int_{0}^{s} \frac{(s-\theta)^{\alpha-1}}{\Gamma(\alpha)} f_{n}(\theta) d \theta d s \\
& -\beta \int_{0}^{T} \int_{0}^{s} \frac{(s-\theta)^{\alpha-1}}{\Gamma(\alpha)} A(\theta) u_{n}(\theta) d \theta d s-\beta \int_{0}^{T} \int_{0}^{s} \frac{(s-\theta)^{\alpha-1}}{\Gamma(\alpha)} f_{n}(\theta) d \theta d s \\
& +\int_{0}^{t} \frac{(t-\theta)^{\alpha-1}}{\Gamma(\alpha)} A(\theta) u_{n}(\theta) d \theta+\int_{0}^{t} \frac{(t-\theta)^{\alpha-1}}{\Gamma(\alpha)} f_{n}(\theta) d \theta
\end{aligned}
$$


We want to prove that there exists $\breve{f} \in S_{F, \breve{u}}^{1}$, such that for all $t \in J$, we get

$$
\begin{aligned}
\check{h}(t)= & \beta \lambda \int_{0}^{T} \int_{0}^{\psi(s)} \frac{(\psi(s)-\theta)^{\alpha-1}}{\Gamma(\alpha)} A(\theta) \check{u}(\theta) d \theta d s+\beta \lambda \int_{0}^{T} \int_{0}^{\psi(s)} \frac{(\psi(s)-\theta)^{\alpha-1}}{\Gamma(\alpha)} \check{f}(\theta) d \theta d s \\
& -\beta \int_{0}^{T} \int_{0}^{\phi(s)} \frac{(\phi(s)-\theta)^{\alpha-1}}{\Gamma(\alpha)} A(\theta) \breve{u}(\theta) d \theta d s-\beta \int_{0}^{T} \int_{0}^{\phi(s)} \frac{(\phi(s)-\theta)^{\alpha-1} \breve{f}(\theta) d \theta d s}{\Gamma(\alpha)} \\
& +\int_{0}^{t} \frac{(t-\theta)^{\alpha-1}}{\Gamma(\alpha)} A(\theta) \check{u}(\theta) d \theta+\int_{0}^{t} \frac{(t-\theta)^{\alpha-1}}{\Gamma(\alpha)} \check{f}(\theta) d \theta .
\end{aligned}
$$

In view of Lemma 1 , define the continuous linear operator $\Phi: L^{1}(J, \mathbb{R}) \rightarrow C(J, \mathbb{R})$ as

$$
\begin{aligned}
\Phi(f)(t)= & \beta \lambda \int_{0}^{T} \int_{0}^{\psi(s)} \frac{(\psi(s)-\theta)^{\alpha-1}}{\Gamma(\alpha)} A(\theta) u(\theta) d \theta d s+\beta \lambda \int_{0}^{T} \int_{0}^{\psi(s)} \frac{(\psi(s)-\theta)^{\alpha-1}}{\Gamma(\alpha)} f(\theta) d \theta d s \\
& -\beta \int_{0}^{T} \int_{0}^{\phi(s)} \frac{(\phi(s)-\theta)^{\alpha-1}}{\Gamma(\alpha)} A(\theta) u(\theta) d \theta d s-\beta \int_{0}^{T} \int_{0}^{\phi(s)} \frac{(\phi(s)-\theta)^{\alpha-1}}{\Gamma(\alpha)} f(\theta) d \theta d s \\
& +\int_{0}^{t} \frac{(t-\theta)^{\alpha-1}}{\Gamma(\alpha)} A(\theta) u(\theta) d \theta+\int_{0}^{t} \frac{(t-\theta)^{\alpha-1}}{\Gamma(\alpha)} f(\theta) d \theta .
\end{aligned}
$$

Thus,

$$
\begin{aligned}
\left|h_{n}(t)-\check{h}(t)\right| \leq & N|\lambda \beta| \int_{0}^{T} \int_{0}^{\psi(s)} \frac{(\psi(s)-\theta)^{\alpha-1}}{\Gamma(\alpha)}\left|u_{n}(\theta)-\check{u}(\theta)\right| d \theta d s \\
& +|\lambda \beta| \int_{0}^{T} \int_{0}^{\psi(s)} \frac{(\psi(s)-\theta)^{\alpha-1}}{\Gamma(\alpha)}\left|f_{n}(\theta)-\check{f}(\theta)\right| d \theta d s \\
& +N|\beta| \int_{0}^{T} \int_{0}^{\phi(s)} \frac{(\phi(s)-\theta)^{\alpha-1}}{\Gamma(\alpha)}\left|u_{n}(\theta)-\check{u}(\theta)\right| d \theta d s \\
& +|\beta| \int_{0}^{T} \int_{0}^{\phi(s)} \frac{(\phi(s)-\theta)^{\alpha-1}}{\Gamma(\alpha)}\left|f_{n}(\theta)-\check{f}(\theta)\right| d \theta d s \\
& +N \int_{0}^{t} \frac{(t-\theta)^{\alpha-1}}{\Gamma(\alpha)}\left|u_{n}(\theta)-\check{u}(\theta)\right| d \theta+\int_{0}^{t} \frac{(t-\theta)^{\alpha-1}}{\Gamma(\alpha)}\left|f_{n}(\theta)-\check{f}(\theta)\right| d \theta .
\end{aligned}
$$

That is, $h_{n}(t) \rightarrow \check{h}(t)$ as $n \rightarrow \infty$. Lemma 1 gives that $\Phi$ o $S_{F, u}^{1}$ is a closed graph operator in $C(J, \mathbb{R}) \times C(J, \mathbb{R})$ and $h_{n} \in \Phi\left(S_{F, u_{n}}^{1}\right)$. Since $u_{n} \rightarrow \check{u}$, then $\breve{h}(t)$ satisfies (34) for some $\check{f} \in S_{F, \check{u}}^{1}$.

Therefore, $Q$ is an upper semicontinuous operator.

Step 4:

Let $\varrho \in(0,1)$ and $u \in \varrho Q u$. Then, there exists $f \in L^{1}(J, \mathbb{R})$ with $f \in S_{F, u}^{1}$, such that, for $t \in J$, we have $h \in Q u$ satisfies (30). As in proving (32), we get

$$
\|h\| \leq \frac{T^{\alpha}(2+\alpha)\left(N\|u\|+\|\varkappa\|_{L_{1}}\right)}{\Gamma(2+\alpha)} .
$$

Thus,

$$
\frac{\Gamma(2+\alpha)\|u\|}{T^{\alpha}(2+\alpha)\left(N\|u\|+\|\varkappa\|_{L_{1}}\right)} \leq 1 .
$$

In view of (27), there exists $\varpi$ such that $\varpi \neq\|u\|$. Set

$$
\Upsilon:=\{u \in C(J, \mathbb{R}):\|u\|<\varpi+1\} .
$$


It is clear that there is no $u \in \partial \Upsilon$ such that $u \in \varrho Q u$ for some $\varrho \in(0,1)$. Further, we get that the operator $Q: \bar{\Upsilon} \rightarrow P_{c p, c v}\left(B_{\sigma}\right)$ is an upper semicontinuous and completely continuous operator.

Consequently, by the nonlinear alternative of Leray-Schauder type, we deduce that $Q$ has a fixed point $u \in \bar{\Upsilon}$ which is a mild solution for Problem (2).

Finally, we give an illustrative example.

Example 1 Consider the following fractional differential inclusion

$$
{ }^{c} D^{0.4}-0.01 \sin u(t) \in\left[\frac{|u|^{3}}{|u|^{3}+3}+4 t^{3}+5, \frac{|u|}{|u|+1}+2 t+1\right] \text { a.e. } t \in[0,1] .
$$

Here, $\alpha=\frac{2}{5}, A(t) u(t)=\frac{1}{100} \sin u(t), N=\frac{1}{100}, T=1, J=[0,1]$ and $F: J \times \mathbb{R} \rightarrow P(\mathbb{R})$ is a set-valued map given by

$$
u \rightarrow F(t, u)=\left[\frac{|u|^{3}}{|u|^{3}+3}+4 t^{3}+5, \frac{|u|}{|u|+1}+2 t+1\right] .
$$

For $f \in F(t, u)$, we have

$$
|f| \leq \max \left\{\frac{|u|^{3}}{|u|^{3}+3}+t^{3}, \frac{|u|}{|u|+1}+1\right\} \leq 10, u \in \mathbb{R} .
$$

Then,

$$
\|F(t, u)\|_{P}=\sup \{|v|: v \in F(t, u), u \in \mathbb{R}\} \leq 10 \text { and }\|\varkappa\|_{L^{1}}=10 .
$$

\section{Consider the deviated-advanced nonlocal condition:}

$$
\sum_{k=1}^{2} 4^{-k} u\left(t_{k}^{2}\right)=\frac{9}{4} \sum_{j=1}^{3} 2^{-j} u\left(\sqrt{t_{j}}\right) .
$$

Clearly, $m=2, n=3, a_{k}=4^{-k}, \quad \sum_{k=1}^{m} a_{k}=\frac{5}{16}, b_{j}=2^{-j}, \quad \sum_{j=1}^{n} b_{j}=\frac{7}{8}, \lambda=\frac{9}{4}$, $\gamma=-\frac{32}{53}, \phi: J \rightarrow J$ is defined by $t \mapsto t^{2}$, and $\psi: J \rightarrow J$ is defined by $t \mapsto \sqrt{t}$.

Applying assumption $\left(H_{5}\right)$, we get $v>27.5321$. Therefore, all conditions of Theorem 3 are satisfied. So, there exists at least one solution for Problem (36) with condition (37).

Now, consider the deviated-advanced nonlocal integral condition:

$$
\int_{0}^{1} u\left(s^{2}\right) d s=\frac{9}{4} \int_{0}^{1} u(\sqrt{s}) d s .
$$

Here, $\lambda=\frac{9}{4}>1$, then $\beta=-\frac{4}{5}$. In view of (27), there exists $\varpi>19.7017$. Therefore, all conditions of Theorem 4 are satisfied. So, there exists at least one solution for problem (36) with the integral condition (38).

Remark 1 For $a_{k}=t_{k}-t_{k-1}, \tau_{k} \in\left[t_{k-1}, t_{k}\right] \subset J$ and $b_{j}=t_{j}-t_{j-1}, \eta_{j} \in$ $\left[t_{j-1}, t_{j}\right] \subset J$, the nonlocal condition for Problem (1) will be $\sum_{k=1}^{m}\left(t_{k}-t_{k-1}\right) u\left(\phi\left(\tau_{k}\right)\right)=$ $\lambda \sum_{j=1}^{n}\left(t_{j}-t_{j-1}\right) u\left(\psi\left(\eta_{j}\right)\right)$. For $u \in C(J, \mathbb{R})$, we have $\lim _{m \rightarrow \infty} \sum_{k=1}^{m}\left(t_{k}-t_{k-1}\right) u\left(\phi\left(\tau_{k}\right)\right)=$ $\lambda \lim _{n \rightarrow \infty} \sum_{j=1}^{n}\left(t_{j}-t_{j-1}\right) u\left(\psi\left(\eta_{j}\right)\right)$ which can be transformed into the nonlocal integral condition $\int_{0}^{T} u(\phi(s)) d s=\lambda \int_{0}^{T} u(\psi(s)) d s$ of Problem (2). 


\section{Funding}

Not applicable.

\section{Availability of data and materials}

The datasets used and/or analyzed during the current study are available from the corresponding author on reasonable request.

\section{Competing interests}

The authors declare that they have no competing interests.

Received: 13 June 2019 Accepted: 11 October 2019

Published online: 09 November 2019

\section{References}

1. Atangana, A.: Fractional Operators with Constant and Variable Order with Application to Geo-Hydrology. Elsevier Science Publishing Co Inc. (2018). https://doi.org/10.1016/C2015-0-05711-2

2. Fallahgoul, H., Focardi, S., Fabozzi, F.: Fractional Calculus and Fractional Processes with Applications to Financial Economics : Theory and Application. Elsevier Science Publishing Co Inc, Academic press, Boston (2017)

3. Hilfer, R.: Applications of Fractional Calculus in Physics. World Scientific, Singapore (2000)

4. Kilbas, A., Srivastava, M., Trujillo, J.: Theory and Application of Fractional Differential Equations. North Holland Mathematics Studies, vol. 204. Elsevier, Amsterdam (2006)

5. Magin, R. L.: Fractional Calculus in Bioengineering. BegellHouse, Connecticut (2006)

6. Podlubny, l.: Fractional Differential Equations. Academic Press, San Diego (1999)

7. Samko, S. G., Kilbas, A. A., Marichev, O. I.: Fractional Integrals and Derivatives, Theory and Applications. Gordon and Breach, Langhorne (1993)

8. Ahmad, B., Ntouyas, S. K.: Existence of solutions for fractional differential inclusions with nonlocal strip conditions. Arab J. Math. Sci. 18, 121-134 (2012)

9. Ahmad, B., Luca, R.: Existence of solutions for sequential fractional integro-differential equations and inclusions with nonlocal boundary conditions. Appl. Math. Comput. 339, 516-534 (2018)

10. Aubin, J., Ceuina, A.: Differential Inclusions: Set-Valued Maps and Viability Theory. Springer-Verlag, Berlin (1984)

11. Benchohra, M., Henderson, J., Ntouyas, S.: Impulsive Differential Equations and Inclusions, Contemp. Math. Appls. Vol.2 (2006)

12. Chang, Y., Nieto, J.: Some new existence results for fractional differential inclusions with boundary conditions. Math. Comput. Model. 49, 605-609 (2009)

13. Goodrich, C. S.: Positive solutions to differential inclusions with nonlocal nonlinear boundary conditions. Appl. Math Comput. 219, 1-11081 (1107)

14. Kisielewicz, M.: Differential Inclusions and Optimal Control. Kluwer, Dordrecht, The Netherlands (1991)

15. Tolstonogov, A. A.: Differential Inclusions in a Banach Space. Kluwer Acad. Publishers, Dordrecht (2000)

16. Wang, J., Ibrahim, A. G., Feckan, M.: Nonlocal Cauchy problems for semilinear differential inclusions with fractional order in Banach spaces. Commun. Nonlinear Sci. Numer. Simul. 27(1-3), 281-293 (2015)

17. Wang, J., Ibrahim, A. G., O'Regan, D., Zhou, Y.: Controllability for noninstantaneous impulsive semilinear functional differential inclusions without compactness. Indag. Math. 29, 1362-1392 (2018)

18. Wu, Z., Min, C., Huang, N.: On a system of fuzzy fractional differential inclusions with projection operators. Fuzzy Sets Syst. 347, 70-88 (2018)

19. Zhou, Y.: Fractional Evolution Equations and Inclusions: Analysis and Control. Elsevier Ltd. (2016). https://doi.org/10. 1016/C2015-0-00813-9

20. Deng, K.: Exponential decay of solutions of semilinear parabolic equations with nonlocal initial conditions. J. Math. Anal. Appl. 179, 630-637 (1993)

21. Boucherf, A: Second-order boundary value problems with integral boundary conditions. 70, 364-371 (2009)

22. Lian, T, Xue, C, Deng, S: Mild solution to fractional differential inclusions with nonlocal conditions. Bound. Value Probl. 219(1) (2016). https://doi.org/10.1186/s13661-016-0724-2

23. El-sayed, A. M. A., Hamdallah, E. M., Elkadeky, Kh. W.: Solutions of a class of deviated-advanced nonlocal problems for the differential inclusion $x^{\prime}(t) \in F(t, x(t))$. Abstr. Appl. Anal. 2011, 1-9 (2011). https://doi.org/10.1155/2011/476392

24. Deimling, K.: Multivalued Differential Equations. Walter De Gruyter, Berlin-New York (1992)

25. Graef, J. R., Henderson, J., Ouahab, A.: Topological Methods for Differential Equations and Inclusions. Taylor \& Francis, CRC Press (2019)

26. Lasota, A, Opial, Z: An application of the Kakutani-Ky-Fan theorem in the theory of ordinary differential equations. Bull. Acad. Polon. Sci. Ser. Sci. Math. Astoronom. Phys. 13, $781-786$ (1955)

27. O'Regan, D: Nonlinear alternatives for multivalued maps with applications to operator inclusions in abstract spaces. Proc. Amer. Math. Soc. 127(12), 3557-3564 (1999)

\section{Publisher's Note}

Springer Nature remains neutral with regard to jurisdictional claims in published maps and institutional affiliations. 